\title{
STRATIFICATION IN WHITE DWARF ATMOSPHERES
}

\author{
DETLEV KOESTER \\ Department of Physics and Astronomy \\ Louisiana State University, Baton Rouge, USA
}

\section{Introduction}

Although the availability of large telescopes and more sensitive detectors has led to a much more complicated picture of white dwarf spectral classes and abundance patterns as compared to the situation ten years ago, it still remains true that the vast majority of white dwarfs show a pure hydrogen spectrum even in temperature regions where the presence of other elements with e.g. solar abundances would be easily revealed.

We have therefore no reason to doubt the general correctness of the idea of diffusion or gravitational separation as originally proposed by Schatzman (1958). Under the combined influence of high gravitational fields and electric forces in the atmospheres and envelopes the heavier elements sink down on a time scale that is short compared to evolutionary time scales for white dwarfs, while the lightest one - usually hydrogen - remains floating to the top. Stellar evolution theory generally predicts the mass of this remaining hydrogen layer to be of the order of $10^{-4}$ solar masses (Iben and Tutukov 1984; Koester and Schönberner 1986; Iben and MacDonald 1985; Mazzitelli and D'Antona 1986; Wood and Faulkner 1986).

Theoretically there are a number of physical processes that could compete with diffusion and possibly prevent the element separation: meridional circulation, mass loss, accretion, radiative levitation, and convection. Under the circumstances encountered in most white dwarfs convection is the most important, although some of the other processes are also invoked in the explanation of certain observations (Fontaine and Wesemael 1987, 1990; Koester 1989a; MacDonald and Vennes 1990). Whenever a stellar region is unstable against convection the macroscopic motions are highly turbulent and element separation is impossible. Only at the bottom (or the top) of this region can diffusion operate.

Convection zones in outer layers do occur only in certain limited regions of effective temperature; outside these regions we should expect a stable, thick hydrogen envelope with a pure hydrogen (DA) spectrum if the star has any significant amount of hydrogen left from the previous evolutionary phases - otherwise it should be a pure helium spectrum.

While this is confirmed by the majority of observations as mentioned above, it is not true for all objects, and it is these exceptions that have been and still are difficult to understand.

i. At the high end of the temperature sequence of hydrogen dominated spectra (50000 to $60000 \mathrm{~K}$ ) the class of DAO objects shows composite spectra with $\mathrm{H}$ dominant but He lines clearly visible.

ii. A number of hot DA white dwarfs, which show only hydrogen in their optical spectra require the presence of helium (or even heavier elements) in their outer layers in order to understand the EINSTEIN and EXOSAT EUV and soft X-ray observations.

iii. Between 15000 and $20000 \mathrm{~K}$ the group of DBA stars show traces of hydrogen in an almost pure helium atmosphere.

iv. Several individual objects outside the above classes show either both $\mathrm{H}$ and $\mathrm{He}$ lines (GD323, a 
DAB) or traces of heavy elements (Feige24, W1346).

In the case of mixed $\mathrm{H} / \mathrm{He}$ spectra - an apparent contradiction to gravitational separation - two alternative explanations are possible:

i. Diffusion has not operated because it was prevented by other processes and the atmosphere is a homogenous mixture of the visible elements.

ii. Diffusion has operated and in fact achieved the equilibrium distribution where the net forces are balanced by the concentration gradient. But the total amount of hydrogen present is so small that the transition from $\mathrm{H}$ to He occurs in the "photosphere" (depending on spectral region, optical or EUV, this can be very different regions geometrically!) and both $\mathrm{H}$ and He remain detectable. Typically the total hydrogen mass would lie in the range $10^{-14}$ to $10^{-18} \mathrm{M}_{\odot}$, extremely small compared to the predictions of standard stellar evolution theory.

In this paper I will not discuss the physical processes competing with diffusion - that has been done elsewhere in many reviews. Instead I will try to review the empirical evidence from an analysis of spectral observations with theoretical model atmospheres using either homogeneous abundances or a stratification of $\mathrm{H} / \mathrm{He}$ as determined from diffusion equilibrium. The question that we are asking is: can the observations distinguish between the two possible interpretations, and if so, which one is favored by the evidence? To keep matters as simple as possible, I will in this talk concentrate on hydrogen dominated atmospheres that show indications of the presence of heavier elements.

\section{Atmosphere Models With Stratified Abundances}

Before I begin with the comparison, I will first discuss briefly the construction of theoretical atmosphere models that include depth dependent abundances as determined from the equilibrium solution of the diffusion equations, and then discuss the observational evidence in view of the above two hypotheses.

The first authors to study chemically inhomogeneous atmosphere models for white dwarfs were Heise and Huizenga (1980), and Muchmore (1982, 1984). The models calculated by Muchmore were later used by Price and Shipman (1985) for a study of hydrogen line profiles. In all these calculations the transition from a pure $\mathrm{H}$ to pure $\mathrm{He}$ layer was treated as a sharp discontinuity. In reality, however, diffusion theory predicts a transition extending over several pressure scale heights, i.e. through the whole atmosphere.

The first systematic study using realistic abundance distributions was performed by Jordan and Koester (1986), who published an extensive grid of synthetic spectra for a large range of effective temperatures and total hydrogen masses. The models are plane parallel atmospheres in LTE, constructed using the Feautrier method with variable Eddington factors as described in more detail in Koester et al. (1979a) and Jordan and Koester (1986). Convection is included in the mixing length approximation, using the Schwarzschild criterium for convective stability. The models are iterated until constancy of the total flux is achieved in all layers to an accuracy of usually $0.1 \%$.

The only difference to our normal atmosphere calculations is that the abundance of hydrogen and helium varies with depth. If we assume that the degree of ionization is constant, the equation of diffusion equilibrium can be solved analytically and the solution gives directly the $\mathrm{H} / \mathrm{He}$ abundance ratio as a function of total pressure (e.g. Dziembowski and Koester 1981). This equation can easily be implemented in atmosphere codes.

Hydrogen is always almost completely ionized in the hot stars considered here. It can also be shown that the abundance profile is not very different for once ore twice ionized helium. Using an effective charge for helium of 2 in the hot models and 1 in the cooler ones should therefore be a good approximation.

If convection zones occur within the atmosphere, they are treated in the following manner: the analytic solution is used above the convection zone. Within the zone the abundances are held constant. If the bottom of the convection zone lies within the atmosphere, the analytic solution for 
the deeper layers is fitted continuously to the abundances in the zone. If convection extends down to the bottom of our atmosphere model, we cannot determine the total hydrogen mass present without an envelope solution with the atmosphere as boundary condition. In that case the $\mathbf{B}$ masses we calculate from the parameter we use (gas pressure at the depth where $\mathrm{H} / \mathrm{He}=1$ ) are only lower limits. For the examples I will use in this paper this does not occur, however. Fig. 1 shows two examples for the abundance distribution as a function of Rosseland optical depth, one in a hot model without convection, and one at a lower temperature with a small convection zone that has no influence on the observable spectra.

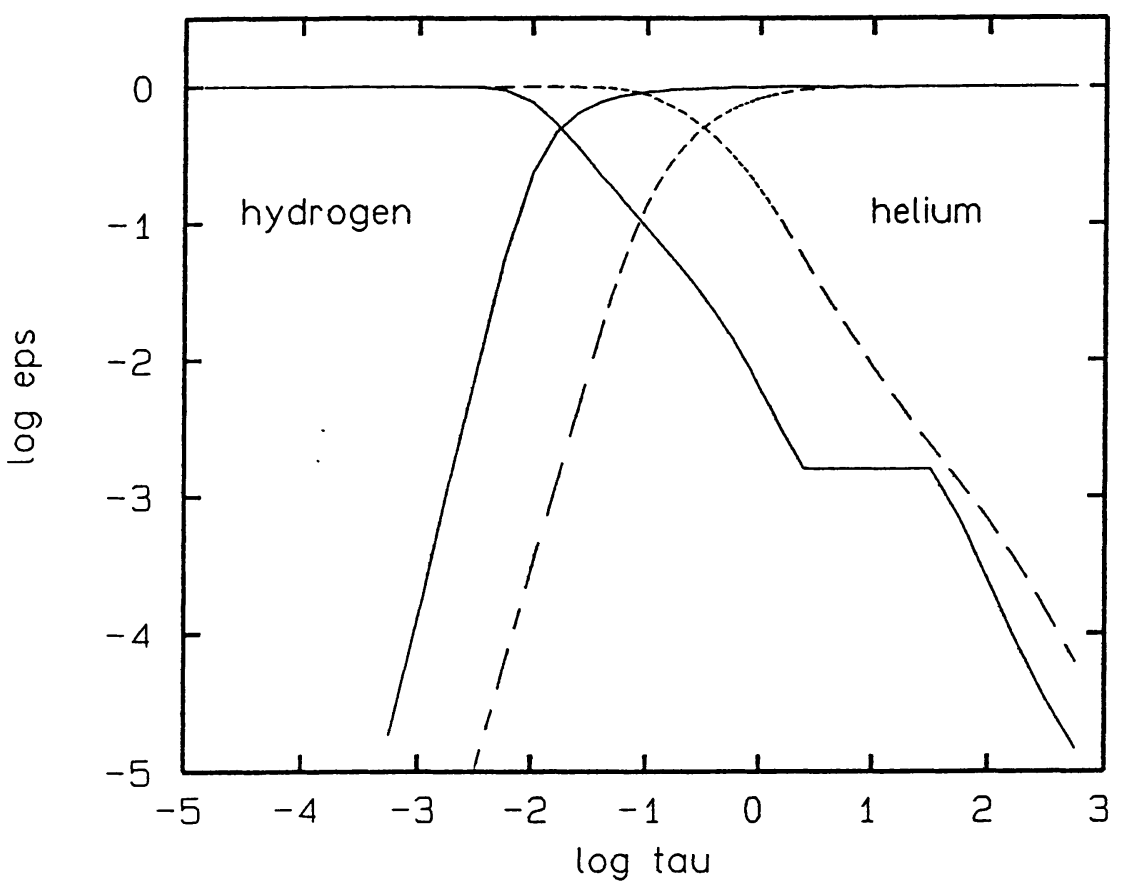

Fig. 1: Abundance distribution in a $60000 \mathrm{~K}, \log \mathrm{g}=8$ model with $\mathrm{M}_{\mathrm{H}}=10^{-16} \mathrm{M}_{\odot}$ (dashed line) and $28000 \mathrm{~K}, \log \mathrm{g}=8, \mathrm{M}_{\mathrm{H}}=510^{-18} \mathrm{M}_{\odot}$ (continuous).

Similar atmosphere models, but extending to larger hydrogen masses as in the grid published by Jordan and Koester (1986), have been constructed by Vennes et al. (1989).

Very recently MacDonald and Vennes (1990) have conducted an extensive study of the interaction of diffusion and convection over the whole range of effective temperatures from 80000 to $10000 \mathrm{~K}$. They solve the equilibrium equations numerically not only for the atmosphere but for the outer envelope in order to determine the extent and change with $T_{\text {eff }}$ of convection zones using different approximations for the convection theory, overshooting, and stability criteria. While this represents a significant progress as far as the general evolution of atmospheric abundances predicted by such models is concerned, they have to make compromises in the treatment of the atmosphere ("grey atmosphere") and detailed calculation of synthetic spectra, which are only used for a qualitative comparison to observations. 


\section{The DAO Stars}

At the hot end of the temperature sequence $(50000$ to $70000 \mathrm{~K})$ one finds in addition to DA with pure hydrogen spectra a small class of stars (the DAO) showing in addition the HeII lines $\lambda 4686$ and 1640, and sometimes a very weak HeI 4471 line.

Detailed atmospheric analyses using homogenous atmospheres have been obtained by Koester et al. (1979b) for the prototype HZ34, and by Wesemael et al. (1985) for the 3 objects known at that time. Holberg et al.(1989) discovered and analyzed He lines in 3 more objects, thus doubling the size of the class. The $\mathrm{He} / \mathrm{H}$ ratios found from these studies cover the range 0.1 to $10^{-3}$.

In a very interesting recent study of low surface brightness (old) planetary nebulae Napiwotzki and Schönberner (1990) found that 4 or 5 out of 12 central stars belong to this class, opening up the possibility that these objects are much more common than previously thought. Their sample clearly shows HeII $\lambda 4686$ of different strengths at about the same effective temperature, very suggestive of the presence of downward diffusion. Final conclusions have to wait for the analysis and the solution of a time scale puzzle: whereas the kinematic age of the nebulae ranges from 30000 to about 100000 yrs the cooling age of a white dwarf of $60000 \mathrm{~K}$ effective temperature is $10^{6}$ years.

A systematic study of DAO spectra using stratified atmospheres has not been done yet. Jordan and Koester (1986), Koester (1989a), and MacDonald and Vennes (1990) demonstrate qualitatively that spectra with $\mathrm{H}$ and HeII lines of the correct strengths can easily be obtained, if the total hydrogen mass is about $10^{-16} \mathrm{M}_{\odot}$. A preliminary analysis of the new objects of Napiwotzki and Schönberner confirms this. Fig. 2 shows one of the new objects (PW1) and two models that approximately reproduce the spectrum: a homogenous atmosphere $(60000 \mathrm{~K}, \mathrm{H} / \mathrm{He}=0.01)$ and a stratified atmosphere $\left(60000 \mathrm{~K}, \mathrm{M}_{\mathrm{H}}=110^{-16} \mathrm{M}_{\odot}\right.$. The models were taken from an available grid with $\log g=8$ and are certainly not the best possible fit. It is obvious, however, that both sets of calculations will to a first approximation be able to fit the spectra. A closer look shows subtle differences: in the stratified model the He line is broader and the hydrogen line narrower, which seems to model the observations slightly better than the homogeneous model. We will, however, have to wait for the final qualitative analysis, before we can use this as an argument for or against the stratification hypothesis.

\section{EUV Observations of DA White Dwarfs}

Observations in the soft X-ray and EUV regions, covering the $228 \AA$ absorption edge of HeII, have so far provided the only opportunity to study the $\mathrm{He} / \mathrm{H}$ abundance ratio in hot DA white dwarfs that show no trace of helium at optical wavelengths.

After the pioneering discoveries of EUV radiation from Sirius B, HZ43, Feige24, and G191-B2B (Mewe et al. 1975a,b; Hearn et al. 1976; Margon et al. 1976a,b; Lampton et al. 1976; Holberg et al. 1980) a more systematic study has been made possible in recent years with the EINSTEIN and EXOSAT satellites. A surprising result of these studies was that a small but finite $\left(\geq 10^{-5}\right)$ amount of helium was necessary in almost all observed DA to explain the EUV count rates (Kahn et al. 1984; Petre et al. 1986; Jordan et al. 1987; Paerels et al. 1987; Paerels 1987; Paerels and Heise 1989).

Another surprise was a trend of increasing He abundance with increasing effective temperature of the white dwarfs found originally by Petre et al. (1986) from their analysis of EINSTEIN data and confirmed by Jordan et al. (1987) from EXOSAT observations of 9 DA. Kahn et al. (1984) proposed two possible mechanisms as explanation for the finite observed He abundances: accretion from interstellar matter and selective radiative acceleration on He ions, supporting them against gravity in the atmospheres. In view of the observed relation between $\mathrm{He} / \mathrm{H}$ and $\mathrm{T}_{\text {eff }}$, Petre et 


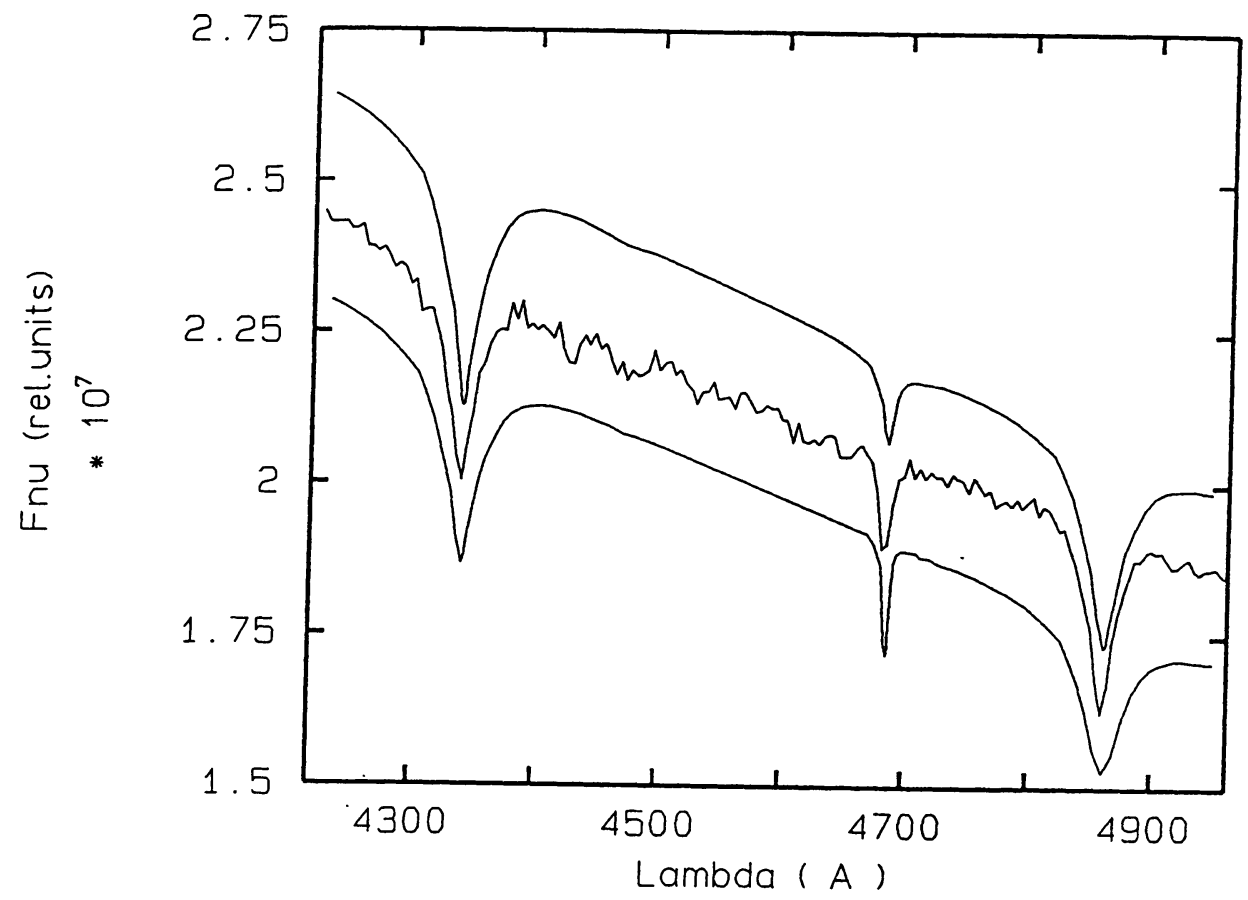

Fig. 2: Observations of the central star of a planetary nebula PW1, a DAO white dwarf, (middle), compared with a stratified (top) and a homogeneous (bottom) atmosphere model. See text.

al. (1986) favored the second process, because only this can naturally lead to such a correlation. However, Vennes et al. (1988) demonstrated that the the amount of helium that can be supported by radiative forces is too small by at least two orders of magnitude to account for the observations.

All abundance determinations in the above mentioned studies were based on the assumption of a homogeneous, mixed $\mathrm{He} / \mathrm{H}$ atmosphere, although as discussed above one would theoretically expect an abundance stratification.

Can chemically stratified atmospheres provide an alternative, satisfactory explanation? The opacity of hydrogen decreases rapidly towards the EUV; it is therefore quite possible that a relatively thick hydrogen layer becomes transparent at short wavelengths and reveals the influence of HeI and HeII absorption edges.

This question was studied by Koester (1989b) and Vennes et al. (1989) using the same set of EXOSAT data. The parameter that was determined from the analysis in these papers is now the total hydrogen mass $\mathrm{M}_{\mathrm{H}}$ instead of the $\mathrm{He} / \mathrm{H}$ abundance ratio. In both papers the conclusion was that the EUV observations can equally well be explained with stratified atmospheres as with homogeneous abundances. The resulting $\mathrm{H}$ masses are in the range from $10^{-16}$ to $10^{-13} \mathrm{M}_{\odot}$.

If both explanations seem equally possible, we still would have no argument for a decision. A closer look, however, shows two arguments that favor the stratification hypothesis. 


\subsection{THE HOT DA G191-B2B}

This DA object shows a pure hydrogen spectrum in the optical and ultraviolet range. Holberg et al. (1989) have determined upper limits for the HeI and HeII lines and drawn attention to the fact that the limit for the He abundance obtained from these limits $\left(2.610^{-4}\right)$ is in sharp contrast with the abundance obtained from the EXOSAT data using homogeneous atmospheres $\left(4.910^{-3}\right.$, Jordan et al. 1987; $3.210^{-3}$, Paerels and Heise 1989). This puzzle is easily solved if we assume a stratified atmosphere: at a total hydrogen mass of $4.710^{-16} \mathrm{M}_{\odot}$ as determined by Koester (1989b) from the EUV data the optical and UV He lines are predicted to be weaker than the limit set by Holberg et al. (1989). Fig. 3 shows the region around HeII $\lambda 4686$ in a new spectrum (Jordan 1990) and the theoretical predictions for the homogeneous and the stratified atmosphere model that both reproduce the EXOSAT data.

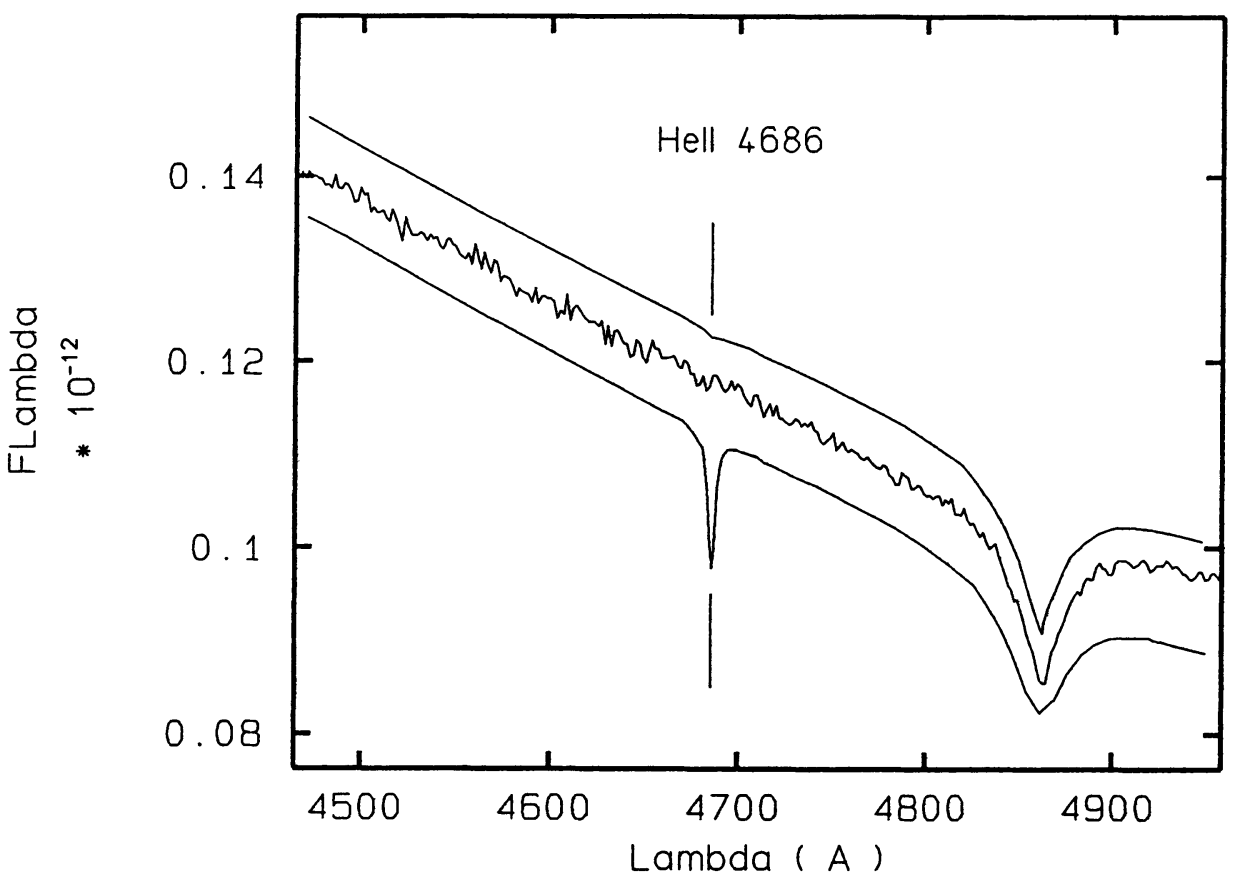

Fig. 3 : Theoretical spectra for stratified (top) and homogeneous (bottom) atmosphere models of G191-B2B that both reproduce the EUV observations, compared to optical observations (middle).

\subsection{THE HOT DA HZ43}

This is the first white dwarf observed in the EUV and soft-X ray region and a primary calibration standard for all experiments at these wavelengths. The EXOSAT observations of this star, however, provide a major puzzle. Paerels and Heise (1989) derive an upper limit of $10^{-5}$ for $\mathrm{He} / \mathrm{H}$ from the EXOSAT spectrum. The EXOSAT broad band filter data are completely incompatible with 
this result if the temperature is confined to the interval obtained from optical and UV observations (Jordan et al. 1987).

If the temperature is lowered to $50000 \mathrm{~K}$, the value favored by Paerels and Heise (1989), the filter data can be explained with a helium abundance of $310^{-6}$, and the discrepancy between EUV spectra and filters disappears (Koester 1989a). But this low effective temperature is now in conflict with other determinations from optical and UV data (57500 K, Holberg et al. 1986).

All constraints can be satisfied by a stratified atmosphere with $\mathrm{T}_{\text {eff }}=54000 \mathrm{~K}$ and $\mathrm{M}_{\mathrm{H}}=1.810^{-14}$ $\mathrm{M}_{\odot}$ (Koester 1989a), again adding some weight in favor of the existence of stratification and very thin $\mathrm{H}$ envelopes.

\section{The DBA Object GD323}

This unique object was originally classified DBp (peculiar) by Greenstein (1969), who noticed weak and sharp He lines. It was found to show broad, shallow hydrogen lines - and thus classified DAB - by Oke et al. (1984) and Liebert et al. (1984).

Using new optical observations and ultraviolet (IUE) spectra, Liebert et al. (1984) demonstrated that this object has an energy distribution from the UV to the red characteristic of a heliumdominated atmosphere around $30000 \mathrm{~K}$, but lines of $\mathrm{H}$ and $\mathrm{He}$ much too weak for this temperature. They analyzed the spectra using homogenous model atmospheres, but were not able to find a consistent fit. After a discussion of all alternatives (interstellar reddening, rotation, accretion disks, binary system) the only hypothesis that survives - as they put it - is the assumption of a stratified atmosphere, which could not be tested at that time due to the lack of detailed models.

A preliminary analysis using stratified atmospheres was presented in Koester (1989a). It indicated the possibility to explain the overall energy distribution as well as the line spectrum with an atmospheric model near the parameters $\mathrm{T}_{\text {eff }}=27000 \mathrm{~K}$ and $\mathrm{M}_{\mathrm{H}}=7.510^{-18} \mathrm{M}_{\odot}$. Since then we (Koester et al. 1990) have used newer and much better optical spectra obtained with the Palomar Double CCD Spectrograph (Greenstein 1986). In addition the IUE spectra used in Liebert et al. (1984) were extracted from the IUE archive, corrected for sensitivity changes with time using a program and data kindly provided by Dr.R.Bohlin and finally corrected to an absolute scale with the white dwarf calibration of Finley et al. (1989).

For the analysis of the observed spectra a new theoretical grid of stratified models and synthetic spectra was calculated for effective temperatures between 26000 and $33000 \mathrm{~K}, \mathrm{M}_{\mathrm{H}} / \mathrm{M}_{\odot}$ between $10^{-16}$ and $10^{-19}$, and $\log g=8$ for most models.

\subsection{THE ENERGY DISTRIBUTION}

With IUE and optical spectra - which fit together very nicely - the energy distribution is known from 1200 to $7000 \AA$. This observed distribution can be fitted with theoretical calculations in the range from $\mathrm{T}_{\text {eff }}=26000$ to $31000 \mathrm{~K}$ (with different total hydrogen masses), because the slope of the energy distribution becomes steeper if the amount of hydrogen in the atmosphere is increased. These fits to the energy distribution define a curve of possible parameter solutions in a $T_{e f f}$ vs. $M_{H}$ plane that leads from relatively "thick" $\mathrm{H}$ layers at $26000 \mathrm{~K}$ to a very thin layer at $31000 \mathrm{~K}$.

\subsection{THE OPTICAL LINE SPECTRUM}

A similar fitting process was carried through with the detailed line spectrum in the optical region from 4000 to $5000 \AA$, again leading to a sequence of possible solution but with the opposite slope: from "thin" $\mathrm{H}$ layers at $26000 \mathrm{~K}$ to "thicker" at $31000 \mathrm{~K}$. Both fit curves therefore define a unique solution near $28000 \mathrm{~K}$ effective temperature.

Fig. 1 shows the comparison for the energy distribution at this effective temperature, Fig. 2 
the optical line spectrum. The agreement between theory and observation is extremely good, the only remaining discrepancy being that the theoretical Balmer lines are slighly narrower than the observed ones.

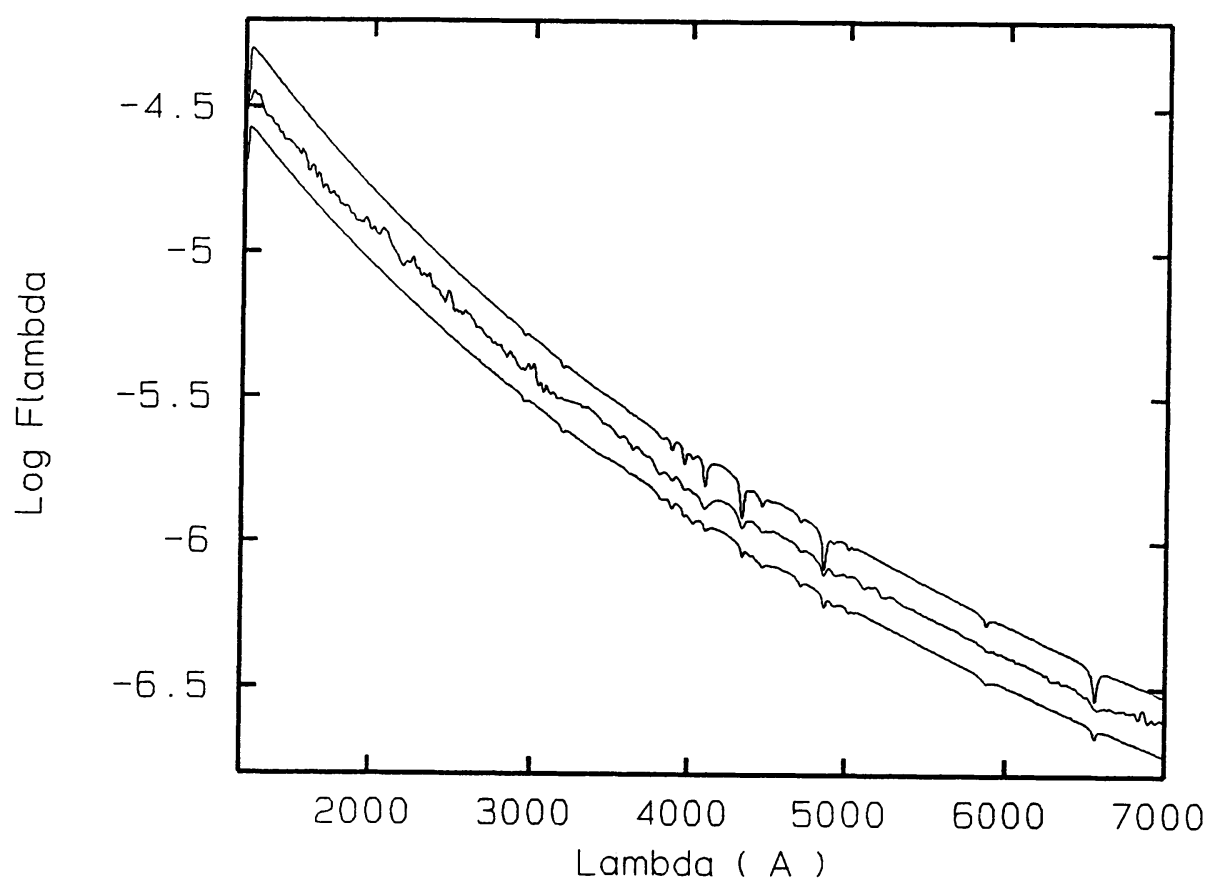

Fig. 4 : Energy distribution of GD323 and models at $28000 \mathrm{~K}, \mathrm{M}_{\mathrm{H}} / \mathrm{M}_{\odot}=3.7$ and $9.210^{-18}$.

The theoretical grid was then expended with much finer stepsizes around the expected solution, the fitting procedure repeated and finally an optimum fit determined with the following parameters:

$$
\mathrm{T}_{\text {eff }}=27500 \pm 500 \mathrm{~K}, \text { and } \mathrm{M}_{\mathrm{H}}=(5.2 \pm 1.0) 10^{-18} \mathrm{M}_{\odot} .
$$

The model atmosphere with these parameters is stable throughout the whole transition zone. A very thin $\mathrm{He}$ convection zone is present in deeper layers, where the composition is almost pure $\mathrm{He}$, and separated from the transition region by at least one pressure scale heigth. We therefore conclude that this is a realistic model for this object.

Our hope to get an even better agreement (broader $\mathrm{H}$ lines) with a higher surface gravity of log $\mathrm{g}=8.5$ was disappointed by the calculations: the observed hydrogen lines do only allow a certain number of hydrogen atoms per $\mathrm{cm}^{-2}$ on top of the atmosphere. A model with higher surface gravity has higher gas pressure at the same optical depth, but the transition from $\mathrm{H}$ to $\mathrm{He}$ has to occur at a lower $\mathrm{P}_{g}$ than in the lower gravity model or else the $\mathrm{H}$ lines become much too strong. At a given equivalent width therefore the lines cannot be made much broader by using higher log $\mathrm{g}$ values.

An alternative explanation for the remaining small discrepancies is the exact structure of the transition profile. As mentioned above, our calculations use a constant degree of ionization for 


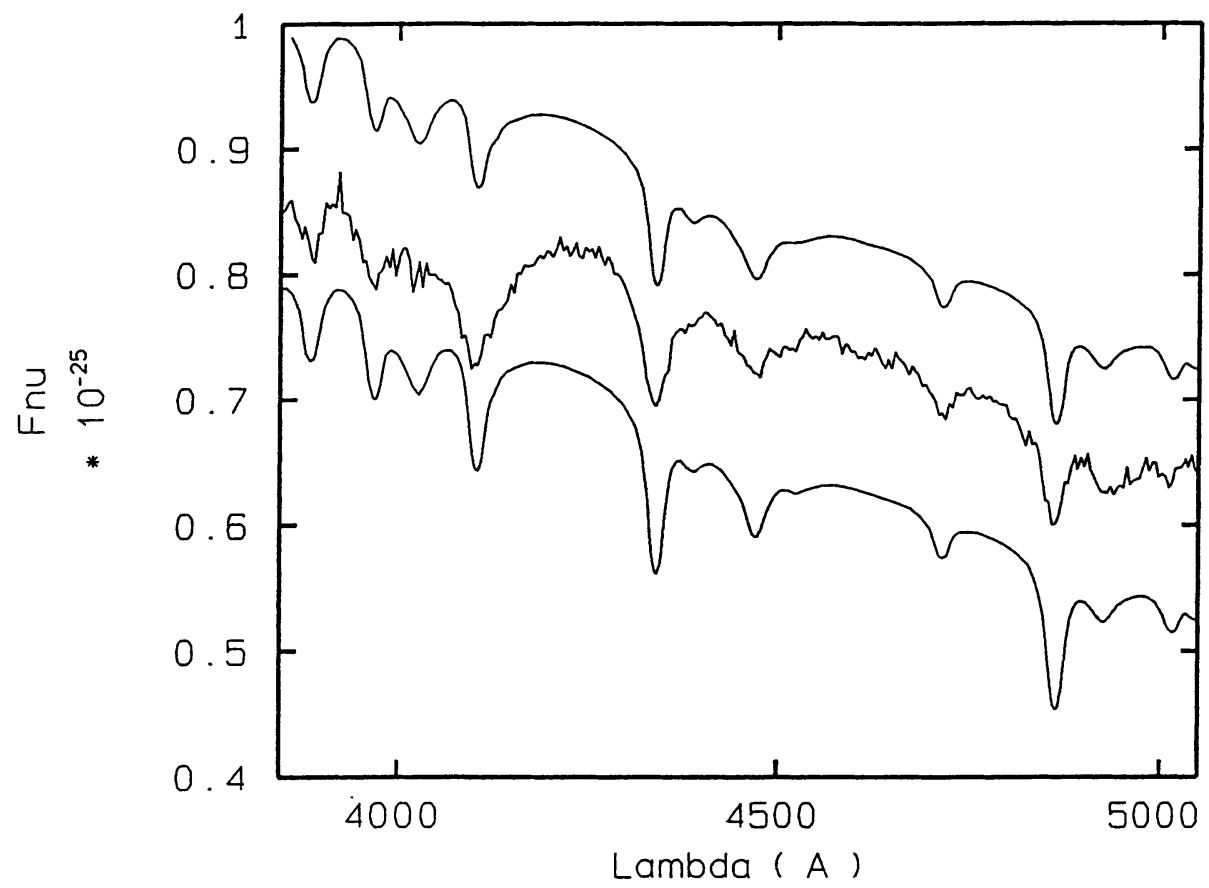

Fig. 5: Optical line spectrum and theoretical models for $28000 \mathrm{~K}, \mathrm{M}_{\mathrm{H}} / \mathrm{M}_{\odot}=4.610^{-18}$ (top) and $5.810^{-18}$ (bottom).

$\mathrm{He}(\mathrm{Z}=1$ in this case) and a ratio of mixing length to pressure scale height of 1 . It is possible that variations of these parameters can change the theoretically predicted abundance profile (e.g. widen the transition zone) and lead to an even better agreement as indicated by some numerical experiments we performed. This would, however, introduce new free parameters into the fitting procedure, and we therefore prefer to keep the simple theoretical model.

In any case the excellent fit with stratified models - as opposed to the complete failure with homogeneous atmospheres - lends strong support to the conclusion that in this object we really observe the result of gravitational settling with an extremely thin hydrogen layer.

How does this object fit into our general picture of the evolution of white dwarf spectral types? With such a thin hydrogen layer the object will have to become a DB at slightly lower effective temperatures, when the strong HeI convection develops and mixes and dilutes the hydrogen to undetectability. It is thus tempting to regard GD323 as an object at or very close to the transition from a DA to a DB, a transition that has to occur around $30000 \mathrm{~K}$, if our current interpretation of the absence of He spectra between 30000 and $45000 \mathrm{~K}$ is correct (Liebert et al. 1987). However, with the same amount of hydrogen as GD323 at higher effective temperatures, a white dwarf would definitely show He lines! A very speculative scenario to solve this puzzle could be the following:

The calculations by MacDonald and Vennes (1990) indicate that a white dwarf with a hydrogen mass of about $10^{-15} \mathrm{M}_{\odot}$ develops a helium convection zone around $\mathrm{T}_{\text {eff }}=28000 \mathrm{~K}$, which contains of the order of $10^{-10} \mathrm{M}_{\odot}$. Even if the total amount of hydrogen would be diluted in this zone, the abundance would be only $10^{-5}$. Our atmosphere calculations indicate that a hydrogen layer 
of order $510^{-18} \mathrm{M}_{\odot}$ on top of this envelope would be stable against convection and thus develop the abundance profile predicted by diffusion equilibrium. At the bottom of our atmosphere the $\mathrm{H}$ abundance is about $10^{-4}$, a convection zone starting much deeper, where this abundance has fallen to e.g. $10^{-5}$ would not change the observable properties of the star, but could increase the total $\mathrm{H}$ mass to $10^{-15} \mathrm{M}_{\odot}$, which would have made this object a DA between 30000 and $45000 \mathrm{~K}$.

\section{The DBA Stars}

About $20 \%$ of the stars with helium dominated atmospheres in the effective temperature range from 14000 to $20000 \mathrm{~K}$ show traces of hydrogen in their spectra (Shipman et al. 1987). Based on analyses with homogeneous atmospheres the $\mathrm{H} / \mathrm{He}$ abundance ratio in these DBA objects is $310^{-5}$ to $410^{-4}$. The transition between radiative and convective zones occurs very high in the photosphere at small optical depths. Using their grey atmosphere synthetic spectra calculations MacDonald and Vennes (1990) estimate a total hydrogen mass for the DBA of $10^{-13}$ to $10^{-12}$, most of which is of course mixed within the deep convection zone. They find that the theoretical hydrogen lines are narrower than observed, which indicates that in the star hydrogen might be present at deeper layers, making the distribution more nearly homogeneous. In their model calculations this can be achieved by including mixing through convective overshooting, leading the authors to the suggestion that eventually the study of DBA stars might be a test for theories of convective overshooting.

In any case, because of the presence of convection zones in the atmosphere and the uncertainty associated with the theory of convection and overshooting it is not very likely that the DBA will provide a useful test for the presence of stratification.

\section{Metals in Hot DA}

In all cases discussed above we were concerned with composite spectra showing $\mathrm{H}$ and $\mathrm{He}$, the elements naturally expected in the outer layers of white dwarfs. There are, however, a few DA that show traces of even heavier metals, especially in the UV (see e.g. Vauclair and Liebert 1987 for a review). A typical example is Feige24, which shows lines of CIV, NV, and SiIV in the photosphere (Dupree and Raymond 1982; Wesemael et al. 1984). These observations do not contradict the diffusion scenario, because it has been shown that the selective radiative forces on these ions can balance gravitation in hot stars (Fontaine and Michaud 1979; Vauclair et al. 1979). These radiation forces depend strongly on the ionization states and these in turn on the temperature; the ions therefore accumulate at specific ranges of optical depth, forming "metal clouds" (Morvan et al. 1986; Chayer et al. 1987). An analysis of the lines in Feige24, using the theoretically predicted stratification of heavy elements (Koester 1989a) leads to mixed results: while the Si and N lines are predicted correctly (within a factor of 2 for the line strengths), the theoretical carbon lines are much stronger than observed.

The observation of small traces of metals has led many authors to suggest that these metals might also be responsible for the EUV absorption necessary to understand the EXOSAT observations, which usually is assumed to be due to helium.

Vennes et al. (1989) have demonstrated that this assumption is in fact a viable alternative for the EUV spectrum of Feige24, although they have to adjust the used elements and their abundances very carefully in order not to violate the observational constraints from the optical and UV spectra. Their result does not eliminate the need for helium as an absorber in other cases. 


\section{Conclusions}

Theoretical reasons as well as the observation that in almost all white dwarfs one element is dominating lead us to believe that diffusion is an important mechanism and is not prevented by competing processes in many cases. In this picture, however, it is difficult to understand the traces of He found in many hot hydrogen-rich white dwarfs (DA and DAO), because diffusion time scales are too short and radiation pressure on helium too small. A possible solution for this puzzle could be to assume that the total amount of hydrogen is very small $\left(\leq 10^{-14} \mathrm{M}_{\odot}\right)$ and that the helium (or metals) are distributed according to diffusion equilibrium, reaching into the visible or EUV photosphere.

All objects showing both $\mathrm{H}$ and $\mathrm{He}$ can at least as well be explained with stratified as with homogeneous atmosphere models. In a few cases where the observations allow a distinction (G191$\mathrm{B} 2 \mathrm{~B}, \mathrm{HZ43}$, GD323) the balance seems to be in favor of stratification. However, the question is not finally settled, and in order to make further progress we will need more studies of the hot DAO and $\mathrm{DO}$, where convection does not complicate the picture, as well as more (preferably spectroscopic!) EUV observations.

Acknowledgements: This work was supported in part by grants from the National Aeronautics and Space Administration (NAG5-990) and from the National Science Foundation (AST-8814711).

\section{References}

Chayer,P., Fontaine,G., and Wesemael,F.: 1989, in White Dwarfs, IAU Coll. No. 114, ed. G.Wegner (Berlin: Springer-Verlag), p.253

Dupree,A.K., and Raymond,J.C. : 1982, Astrophys.J. (Letters) 263, L63

Dziembowski,W., and Koester,D.: 1981, Astron. Astrophys. 97, 16

Finley,D., Basri,G., and Bowyer,S.: 1989, in White Dwarfs, IAU Coll. No.114, ed. G.Wegner

(Berlin: Springer-Verlag), p.139

Fontaine,G., and Michaud,G.: 1979, Astrophys.J. 231, 826

Fontaine,G., and Wesemael,F.: 1987, in The Second Conference on Faint Blue Stars, IAU Coll. No.95, eds. A.G.D.Philip, D.S.Hayes, and J.Liebert (Schenectady: L.Davis Press), p. 319

Fontaine,G., and Wesemael,F.: 1990, these proceedings

Greenstein,J.L.: 1969, Astrophys.J. 158, 281

Greenstein,J.L.: 1986, Astrophys.J. 304, 334

Hearn,D.R., Richardson,J.A., Bradt,H.V.D., Clark,G.W., Lewin,W.H.G., Mayer,W.F., McClintock,J.E., Primini,F.A., and Rappaport,S.A. : 1976, Astrophys.J. (Letters) 203, L21

Heise,J., and Huizenga,H.: 1980, Astron. Astrophys. 84, 280

Holberg,J.B., Sandel,B.R., Forrester,W.T., Broadfoot,A.L., Shipman,H.L., and Barry,J.L.: 1980, Astrophys.J. (Letters) 242, L119

Holberg,J.B., Wesemael,F., and Basile,J.: 1986, Astrophys.J. 306, 629

Holberg,J., Kidder,K., Liebert,J., and Wesemael,F.: 1989, in White Dwarfs, IAU Coll. No.114, ed. G. Wegner (Berlin: Springer-Verlag), p.188

Iben, I.Jr., and MacDonald, J.: 1985, Astrophys.J. 296, 540

Iben, I.Jr., and Tutukov, A.V.: 1984, Astrophys.J. 282, 615

Jordan,S.: 1990, private communication

Jordan,S., and Koester,D.: 1986, Astron. Astrophys. Suppl. 65, 367

Jordan,S., Koester,D., Wulf-Mathies,C., and Brunner,H.: 1987, Astron. Astrophys. 185, 253

Kahn,S., Wesemael,F., Liebert,J., Raymond,J., Steiner,J., and Shipman,H.L. : 1984, Astrophys.J. 278, 255

Koester,D.: 1989a, in White Dwarfs, IAU Coll. No.114, ed. G.Wegner (Berlin: Springer-Verlag), p.206 
Koester,D.: 1989b, Astrophys.J. 342, 999

Koester,D., Liebert,J., and Greenstein,J.L.: 1990, in preparation

Koester,D., Liebert,J., and Hege, E.K.: 1979b, Astron. Astrophys. 71, 163

Koester, D., and Schönberner, D.: 1986, Astron. Astrophys. 154, 125

Koester,D., Schulz,H., and Weidemann,V.: 1979a, Astron. Astrophys. 76, 262 Astrophys. 76,262

Lampton,M., Margon,B., Paresce,F., Stern,R., and Bowyer,S. : 1976, Astrophys.J. (Letters) 203, L71

Liebert,J., Wesemael,F., Sion,E.M., and Wegner,G.: 1984, Astrophys.J. 277, 692

Liebert,J., Fontaine,G., and Wesemael,F.: 1987, Mem. Soc. Astron. Italiana 58,17

MacDonald,J., and Vennes,S.: 1990, preprint

Margon,B., Malina,R., Bowyer,S., Stern,R., and Paresce,F. : 1976a, Astrophys.J. (Letters) 203, L25

Margon,B., Lampton,M., Bowyer,S., Stern,R., and Paresce,F. : 1976b, Astrophys.J. (Letters) 210, L79

Mazzitelli, I., and D'Antona, F.: 1986, Astrophys.J. 308, 706

Mewe,R., Heise,J., Gronenschild,E.H.B.M., Brinkman,A.C., Shriver,J., and den Boggende,A.J.F.: 1975a, Nature 256,711

Mewe,R., Heise,J., Gronenschild,E.H.B.M., Brinkman,A.C., Shriver,J., and den Boggende,A.J.F.: 1975b, Astrophys.J. (Letters) 202, L67

Morvan,E., Vauclair,G., Vauclair,S.: 1986, Astron. Astrophys. 163, 145

Muchmore,D.: 1982, Astrophys.J. 259, 749

Muchmore,D.: 1984, Astrophys.J. 278, 769

Schönberner,D. and Napiwotzki,R: 1990, private communication

Oke,J.B., Weidemann,V., and Koester,D.: 1984, Astrophys.J. 281, 276

Paerels,F.B.S., and Heise,J.: 1989, Astrophys.J. 339, 1000

Paerels,F.B.S., Heise,J., Kahn,S.M., and Rogers,R.D. : 1987, Astrophys.J. 322, 315

Paerels,F.B.S.: 1987, Thesis, University of Utrecht

Petre,R., Shipman,H.L., and Canizares,C.R.: 1986, Astrophys.J. 304, 356

Price,C.W., and Shipman,H.L.: 1985, Astrophys.J. 295, 561

Schatzman,E.: 1958, White Dwarfs, (Amsterdam: North Holland)

Shipman,H.L., Liebert,J, and Green,R.F.: 1987, Astrophys.J. 315, 239

Vauclair,G., Vauclair,S., and Greenstein,J.L.: 1979, Astron. Astrophys. $\quad 80,79$

Vennes,S., Fontaine,G., and Wesemael,F.: 1989, in White Dwarfs, IAU Coll. No.114, ed. G.Wegner (Berlin: Springer), p.368

Vennes,S., Pelletier,C., Fontaine,G., and Wesemael,F.: 1988, Astrophys.J. 331, 876

Vennes,S., Chayer,P., Fontaine,G., and Wesemael,F.: 1989, Astrophys.J. 336, L25

Wesemael,F., Green,R.F., and Liebert,J.: 1985, Astrophys.J. Suppl. 58, 379

Wesemael,F., Henry,R.B.C., and Shipman,H.L.: 1984, Astrophys.J. 287, 868

Wood, P.R., and Faulkner, D.J.: 1986, Astrophys.J. 307, 659 